\title{
Recherches expérimentales
}

\section{sur le cycle biologique d'Opistborchis chabaudi n. sp. Description de l'adulte}

\author{
par R. BOURGAT et S.-D. KULO \\ Laboratoire de Parasitologie, Université du Bénin, B.P. 1515, Lomé, Togo.
}

\section{Résumé.}

$O$. chabaudi n. sp. est obtenu expérimentalement à partir de cercaires, produites par des rédies, et émises naturellement par le Mollusque prosobranche Gabbia neumanni (Martens, 1898). La cercaire s'enkyste chez plusieurs têtards d'Amphibiens. L'adulte se développe dans les voies biliaires du Chat domestique. $O$. chabaudi se caractérise essentiellement par sa silhouette, ainsi que par la forme et la situation de ses appareils reproducteurs. La possibilité d'infestation humaine accidentelle n'est pas écartée.

\section{Summary.}

Experimental research on the life cycle of Opisthorchis chabaudi n. sp. Description of the adult.

$O$. chabaudi n. sp. is experimentally obtained from cercariae, born into rediae and naturally produced by the snail Gabbia neumanni (Martens, 1898). Cercariae encyst into some tadpoles. The adulte grows into the biliary ducts of the Cat. $O$. chabaudi is mainly characterized by its outline, therefore by the shape and position of its reproductive systems. Accidental human contamination possibility is not denied.

Reçu le 21 juin 1977. 


\section{Introduction}

Une cercaire ocellée, émise par le Mollusque prosobranche Gabbia neumanni (Martens, 1898) s'enkyste expérimentalement chez plusieurs espèces de tétards. d'Amphibiens anoures. Les métacercaires se développent chez le Chat domestique: où elles évoluent en une nouvelle espèce de Douve hépatobiliaire dont nous donnons la description ci-après.

\section{I. - Description d'Opisthorchis chabaudi n. sp. (fig. 1)}

Hôte eXPÉRIMENTAL: Chat domestique.

Habitat : voies biliaires.

Localité: Kovié (Togo).

\section{Morphologie.}

Le corps $(5,5 \mathrm{~mm} \times 2 \mathrm{~mm})$, régulièrement allongé, est aminci antérieurement: et postérieurement arrondi. Le tégument est finement spinulé. L'acétabulum (diamètre : $140 \mu$ ) est situé à $1,25 \mathrm{~mm}$ de l'extrémité antérieure et à $4,12 \mathrm{~mm}$ de l'extrémité postérieure, soit au quart de la longueur.

\section{Anatomie.}

La ventouse orale $(177 \mu \times 135 \mu-$ V.O./V.V. $=1,11)$ s'ouvre dans un pharynx $(118,5 \mu \times 139,5 \mu)$ plus large que long, qui est suivi d'un court œsophage $(250 \mu)$. La bifurcation caecale est sensiblement au tiers de la distance séparant les deux ventouses. Les caecums atteignent l'extrémité postérieure; ils sont réguliers, sauf dans leur partie terminale, au niveau des glandes génitales où leur bord externe devient alors lobé ou festonné.

La vessie excrétrice passe entre les deux testicules, elle contourne l'antérieur par la droite, le postérieur par la gauche et s'ouvre à l'extrémité postérieure du corps.

Les testicules (antérieur $=160 \mu \times 212 \mu$, postérieur $=220 \mu \times 168 \mu$ ) sont. en tandem, très légèrement obliques, inter-caecaux dans la cinquième partie postérieure du corps. Chacun est formé de trois à cinq lobes parfois subdivisés en lobules. Le canal efférent du testicule postérieur circule par la droite, celui du testicule antérieur par la gauche. La vésicule séminale est bien visible dans sa partie terminale; elle contourne l'acétabulum par la droite et s'ouvre au pore génital immédiatement anté-acétabulaire.

L'ovaire $(441 \mu \times 135 \mu)$, situé aux trois quarts du corps, est disposé horizontalement. Il se compose de deux groupes de trois ou quatre digitations latérales réunies par un isthme médian. Il coiffe directement le réceptacle séminal volumineux $(306 \mu \times 177 \mu)$, ovoïde, situé en oblique sur la droite du corps. Cet organe pré- 
cède immédiatement le testicule antérieur. Les vitellogènes s'étendent d'un niveau à peine inférieur à celui du bord postérieur de l'acétabulum jusqu'au niveau de l'ovaire, en deux bandes extracaecales très rarement interrompues. Les follicules de la région postérieure peuvent être détachés du reste de la colonne; ils sont drainés par une branche particulière du vitelloducte longitudinal. Les branches antérieure et postérieure du réseau vitelloducte d'un même côté confluent dorsalement au caecum pour donner naissance au vitelloducte transverse. Les boucles utérines occupent l'espace intracaecal du voisinage de l'ovaire jusqu'à proximité de l'acétabulum qu'elles contournent par la gauche pour atteindre le pore génital anté-acétabulaire. L'importance de la zone utérine (approximativement la distance acétabulum-ovaire) représente la moitié de la longueur totale. Les coudes utérins peuvent se poser exceptionnellement sur la face ventrale des caecums, sans les déborder, ni même les masquer exagérément. Les œufs, operculés, ornés d'un petit mucron postérieur, mesurent après émission de 26 à $32 \mu \times 12$ à $16 \mu$. Les mensurations prises sur des éléments utérins sont légèrement inférieures.

\section{Discussion.}

Par l'ensemble de ses caractères, ce Ver rentre dans le genre Opisthorchis Blanchard, 1895. Il se sépare en particulier de Clonorchis Looss, 1907, dont les testicules, ramifiés, débordent largement les caecums, et dont la vessie excrétrice dorsale aux testicıiles est pratiquement rectiligne.

Le seul Opisthorchis signalé dans la région $(O$. lomeensis Bourgat et Combes, 1975), parasite l'Amphibien Aubria subsigillata (Duméril, 1856). Les deux vers diffèrent largement l'un de l'autre par la forme du corps, les dimensions, le rapport ventousaire, l'extension des vitellogènes, la forme des testicules et de l'ovaire.

A l'heure actuelle (Yamaguti, 1971), 5 espèces d'Opisthorchis de Mammifères sont connues, dont une seule d'Afrique: O. starkovi Biocca et Benetti, 1956, livré au jardin zoologique de Rome par un Serval originaire de Somalie.

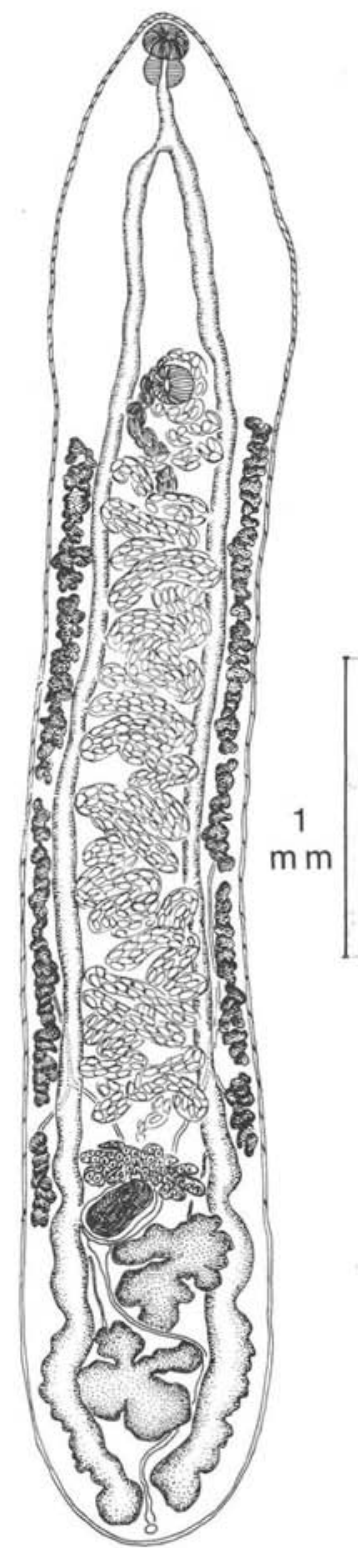

FIG. 1. - Opisthorchis chabaudi n. sp., vue ventrale. 
O. chabaudi se sépare d'O. felineus (Rivolta, 1884) par sa forme allongée (lancéolée chez $O$. felineus), les dimensions moindres ( $6 \mathrm{~mm}$ au lieu de $12 \mathrm{~mm}$ ), la plus grande longueur de l'œsophage qui est très réduit chez $O$. felineus, la forme de l'ovaire (digitée au lieu de simple ou très légèrement lobée) et sa situation aux trois quarts du corps chez $O$. chabaudi, dans le deuxième tiers chez $O$. felineus, les testicules plus profondément lobés, la position du réceptacle séminal post-ovarien au lieu de paraovarien chez $O$. felineus.

O. chabaudi se sépare d'O. tenuicollis (1) (Rudolphi, 1819) par la forme de l'ovaire, digitée au lieu de sub-ovale, la lobation des testicules beaucoup plus profonde, et la disposition des vitellogènes dont les follicules forment une bande serrée très rarement interrompue, au lieu d'un chapelet d'îlots individualisés chez O. tenuicollis.

$O$. chabaudi diffère d'O. tonkae Wallace et Penner, 1939, par la moindre étendue des vitellogènes qui, chez $O$. tonkae, s'étendent d'un niveau antérieur à l'acétabulum, jusqu'au testicule postérieur, l'œsophage mieux développé, les testicules plus profondément lobés, l'ovaire digité au lieu d'entier chez $O$. tonkae.

$O$. chabaudi diffère d'O. viverrini (Poirier, 1886) par la forme allongée de son corps aux bords parallèles, alors qu'O. viverrini est nettement lancéolé, la disposition des vitellogènes formant chez $O$. viverrini comme chez $O$. tenuicollis, un chapelet d'îlots individualisés, la forme de l'ovaire, arrondie ou peu lobée chez $O$. viverrini; enfin, chez ce Ver, la zone utérine représente de 0,36 à 0,40 de la longueur totale, au lieu de la moitié chez $O$. chabaudi.

$O$. chabaudi diffère par sa forme, d'O. starkovi qui est lancéolé, et aussi par les vitellogènes plus étendus vers l'avant, le trajet de la vessie excrétrice qui, chez $O$. starkovi passe dorsalement aux testicules au lieu de les contourner (2), le rapport ventousaire ( $1,11 \mathrm{chez} O$. chabaudi, $1,14 \mathrm{chez} O$. starkovi), l'importance de la zone utérine (moitié du corps chez $O$. chabaudi, 39 pour cent chez $O$. starkovi) et les dimensions des œufs, légèrement plus petits chez $O$. starkovi (16 à $22 \mu \times 8$ à $11 \mu)$.

L'analyse qui précède montre l'opportunité de créer, pour l'Opisthorchis développé, au Togo, dans les voies biliaires d'un Chat domestique, l'espèce $O$. chabaudi (3) n. sp., dédiée à M. le $\mathrm{P}^{r}$ A.-G. Chabaud, Directeur du laboratoire des Vers au Muséum de Paris. La diagnose est la suivante: corps allongé et non lancéolé, tégument discrètement spinulé, acétabulum au quart de la longueur, zone utérine étendue sur la moitié de la longueur totale, testicules profondément lobés, ovaire digité, vitellogènes disposés en deux bandes extracaecales régulières, rarement interrompues, d'un niveau immédiatement postérieur à l'acétabulum jusqu'à l'ovaire.

$O$. chabaudi est le deuxième Opisthorchis de Mammifère signalé en zone éthiopienne et le deuxième représentant du genre pour l'Afrique occidentale.

(1) O. tenuicollis (Rudolphi, 1819) englobe O. felineus pour plusieurs auteurs: Wallace et Penner 41939), Euzeby (1971); par contre Yamaguti (1971), Dawes (1968), ne suivent pas cette assimilation.

(2) Pour Yamaguti (1971), ce caractère est plutôt propre au genre Clonorchis.

(3) Type déposé dans les collections du Muséum de Paris sous le $\mathrm{n}^{\circ} 833$ TA. 


\section{II. - Cycle biologique expérimental d'O. chabaudi n. sp.}

\section{Résumé du cycle.}

L.e premier hôte intermédiaire naturel est, au Togo, le Mollusque prosobranche Gabbia neumanni où se développe une seule génération de rédies. La cercaire s'enkyste expérimentalement chez plusieurs espèces de tétards d'Amphibiens anoures. La maturation de la métacercaire dure au maximum trente-cinq jours. Au bout de cette période, ingérée expérimentalement par un Chaton domestique, la métacercaire peut évoluer et donner, moins de sept semaines plus tard, l'adulte ovigère. Le miracidium et le sporocyste n'ont pu être observés.

L'originalité de ce cycle d'Opisthorchis réside essentiellement dans l'utilisation: de tétards comme deuxième hôte intermédiaire.

\section{LA RÉDIE ( $f$ ig. $2 a$ et $b$ ).}

La rédie, formée naturellement chez G. neumanni, est très allongée (1440 $\mu \times$ $90 \mu$ ), pourvue d'un pharynx important $(18,5 \mu \times 20,3 \mu)$, mais d'un caecum digestif réduit $(30 \mu)$ contenant une matière brunâtre. La rédie renferme de 30 à 40 cercaires à différents stades de maturité.

\section{La cercaire ( $f$ ig. $2 c$ et $d$ ).}

Les cercaires, libérées prématurément par la rédie achèvent leur maturation dans la glande digestive du Mollusque.

La cercaire nageante, après fixation au formol tiède à 1 pour cent, mesure $470 \mu$ de longueur totale. Le corps cercarien $(174 \mu \times 57 \mu)$ est recouvert d'une spinulation forte antérieurement, mais atténuée vers la région postérieure. La ventouse orale $(33 \mu \times 24 \mu)$, protractile est armée de 16 à 18 fortes épines. Le pharynx, aussi long que large mesure $9 \mu$. Les ocelles, de forme circonflexe, sont toujours: flagrants. Les glandes de pénétration sont comptées au nombre de 6 paires, mais leur observation est gênée par l'abondance de glandes kystogènes. L'acétabulum n'est. pas apparent. La vessie excrétrice, dont la paroi est constituée de grandes cellules est en forme d'étrier. Les canaux collecteurs s'abouchent aux angles latéraux antérieurs de la vessie; les pores excréteurs s'ouvrent au $1 / 5^{\mathrm{e}}$ de la longueur de la queue. La queue $(300 \mu)$, est claire et striée transversalement. La membrane natatoire, large, entoure la partie terminale de la queue, mais s'étend inégalement sur les deux bords.

L'activité de la cercaire est entrecoupée de périodes d'immobilité pendant lesquelles, le corps dirigé vers le bas, incurvée, elle se laisse déposer sur le fond du récipient. C'est au cours d'un de ces temps de repos qu'elle peut rencontrer le deuxième hôte intermédiaire, lui-même immobilisé sur le fond, auquel elle adhère alors, avant de pénétrer à travers la peau. 


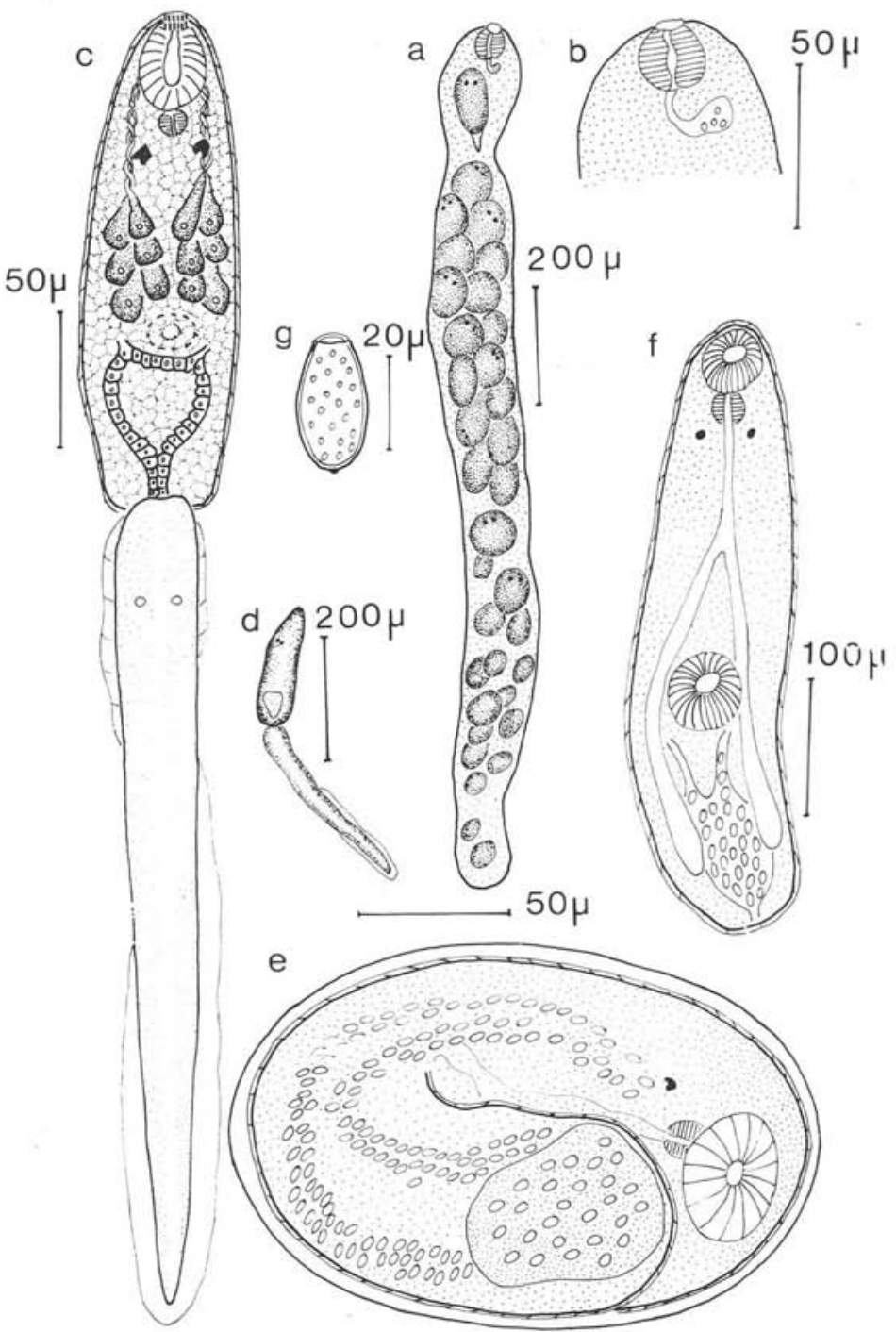

Fig. 2. - $a:$ rédie ; $b$ : rédie, détail de la région antérieure ; $c$ : cercaire; $d:$ cercaire, position d'immobilité; $e$ : kyste métacercarien; $f:$ métacercaire âgée de 35 jours dékystée; g: œuf émis.

Expérimentalement, la cercaire d'O. chabaudi s'enkyste chez les différents têtards qui lui ont été présentés: Bufo regularis Reuss, 1834, Hylarana albolabris (Hallowell, 1856) et Conraua derooi Hulselmans, 1971. L'enkystement n'a pu être obtenu jusqu'ici .chez plusieurs espèces d'alevins. 
LA MÉtACERCAire ( $f i g .2 e$ et $f$ ).

Les kystes métacercariens $(200 \mu \times 125 \mu)$ sont transparents à l'état jeune mais deviennent rapidement opaques.

Les métacercaires âgées de trente-cinq jours, dékystées in vitro, mesurent $478 \mu$ $\times 113 \mu$. Elles sont spinulées, ocellées et présentent un bel acétabulum (diamètre: $52 \mu)$ ainsi que de longs cæcums digestifs; leur vessie excrétrice est bourrée de granules réfringents.

Un Chaton non sevré, nourri par trois têtards d'H. albolabris porteurs de métacercaires de cet âge-là, a livré, à l'autopsie, sept semaines plus tard, deux $O$. chabaudi localisés dans les voies biliaires. La vésicule biliaire retenait une quantité importante d'œufs (fig. $2 \mathrm{~g}$ ) mesurant $30 \mu$ de long environ.

Par contre, plusieurs Amphibiens, des Agames, un Lapereau et un Rat blanc, infestés dans les mêmes conditions, se sont révélés négatifs; deux cobayes, ayant subi des infestations identiques, sont morts au bout de dix-sept et vingt-cinq jours sans que la dissection ne montre la présence d'Opisthorchis, même immatures.

\section{Conclusion}

Le développement d'O. chabaudi, obtenu expérimentalement à partir de cercaires naturelles, suit le schéma des cycles biologiques d'Opisthorchis, à savoir: pénétration de la cercaire à travers la peau du deuxième hôte intermédiaire aquatique et évolution en adulte de la métacercaire dans les voies biliaires de l'hôte définitif. Son originalité réside dans l'utilisation du Têtard, précisément comme deuxième hôte intermédiaire.

Il reste assurément à rechercher si ce rôle n'est pas, dans la nature, tenu par un Poisson, comme dans les autres cycles d'Opisthorchis connus, ou s'il se confirme, comme l'indique l'expérience, que le deuxième hôte intermédiaire habituel soit bien un Têtard.

Dans la première hypothèse - celle du vecteur Poisson —, la période d'infestation du deuxième hôte est brève car la présence de poissons dans l'endémiotope, liée à la présence de l'eau provenant d'une branche du fleuve Sio en période de crue, est fugace. Cette limitation serait compensée par une diffusion efficace des métacercaires par les poissons, dont la dispersion dans le bassin inférieur du Sio est certainement très large.

Dans la deuxième hypothèse - celle, conforme à l'expérience, du vecteur Têtard -, la période d'infestation du deuxième hôte est plus étalée dans le temps, car il suffit de fortes pluies locales pour qu'apparaissent des mares où coexistent Mollusques et têtards, mais d'où les poissons sont absents. Compte tenu de la plus grande concentration de têtards, le nombre de vecteurs atteints est donc vraisemblablement plus élevé que lorsqu'il s'agit de poissons. La population d'Opisthorchis serait alors limitée par la moindre dispersion de l'agent infestant, car les Anoures se déplacent, malgré tout, moins que les poissons. 
Ces deux voies de diffusion des métacercaires ne sont pas exclusives et il est tout à fait envisageable que le parasite puisse les emprunter indifféremment pour maintenir sa fréquence.

\section{Conclusion générale}

$O$. chabaudi n. sp. est le deuxième représentant du genre, signalé au Togo, dans le même biotope, après $O$. lomeensis parasite d'Amphibien.

L'hôte définitif reste à définir avec certitude; il s'agit très vraisemblablement d'un Mammifère, ce qui laisse une possibilité d'infestation humaine accidentelle. Si, comme l'indique l'expérience, le deuxième hôte intermédiaire est un Têtard, le risque est réduit, les habitants de la région ne consommant pas d'Amphibiens; mais si, comme dans les autres cycles d'Opisthorchis, le vecteur habituel des métacercaires est un Poisson, alors, en toute logique, le danger de contamination des populations humaines existe réellement.

\section{Bibliographie}

BIocca (E.) et BeNETtI (M.), 1956. - Opisthorchis starkovi n. sp. parassita di Felis serval. Atti Acad. naz. Lincei-Rend. cl. Sc. fis. mat. et nat. Rome, sér. 8, 21, 456-460.

Blanchard (R.), 1895. - Animaux parasites. Bull. Soc. zool. France, 20, 217.

Bourgat (R.) et Combes (C.), 1975. - Opisthorchis lomeensis n. sp., Douve biliaire de l'Amphibien Aubria subsigillata au Togo. Ann. Parasit. hum. comp., 50, 297-301.

Dawes (B.), 1968. - The Trematoda. Cambridge University Press, édit., New York, 644 p.

EuZÉBY (J.), 1971. - Les maladies vermineuses des animaux domestiques et leurs incidences sur la pathologie humaine. II. Maladies dues aux Plathelminthes: Trématodes, livre 1. Vigot, édit. Paris, 798 p.

Poirier (J.), 1886. - Trématodes nouveaux ou peu connus. Bull. Soc. philom. Paris, sér. 7, 10, 20-40.

Wallace (F.) et Penner (L.), 1939. - A new liver fluke of the genus Opisthorchis. J. Parasitology, 25, 437-439.

Yamagutı (S.), 1971. - Synopsis of digenetic Trematodes of Vertebrates, Vol. I et II, Keigaku Publi. Comp., Tokyo, 1074 p. et 349 p. 